\title{
Is computed tomography of the brain necessary in patients with clinically suspected depressed skull fracture and no focal neurological deficit?
}

\author{
J A Rabie, $M B$ ChB \\ S Otto, MB ChB, MMed (Diagnost Radiol) \\ A J le Roux, MB ChB \\ Department of Diagnostic Radiology, Faculty of Health Sciences, University \\ of the Free State, Bloemfontein
}

\begin{abstract}
Objective. The objective of the study was to determine whether computed tomography (CT) of the brain is necessary in all head trauma patients with clinically suspected depressed skull fractures, Glasgow Coma Scale (GCS) scores of 13 and above, and no focal neurological deficits.

Design. A retrospective descriptive analysis was undertaken of patients of all ages who presented at the trauma unit of the Pelonomi Hospital Complex in Bloemfontein with GCS of 13 to 15, depressed skull fracture, no clinical focal neurological deficit, and who also underwent CT of the brain. Data were obtained from patients' files, and radiological reports and were analysed by the Department of Statistics, University of the Free State.

Results. One hundred and thirty-one patients were included in the study, of whom $56(42.7 \%)$ were found to have substantial intracranial pathology as determined by CT. Twenty-four (18.3\%) of these patients had a GCS of 13, of whom 6 (25\%) had normal CT scans and 18 (75\%) intracranial pathology. Twenty-eight $(21.37 \%)$ of the 56 patients with intracranial pathology had a GCS of 14, of whom 11 (39.3\%) had normal CT scans and 17 (60.7\%) intracranial pathology. A GCS of 15 was determined in $79(60.3 \%)$ of the 131 patients, of whom $58(73.4 \%)$ had normal CT scans and 21 (26.6\%) intracranial pathology.

Conclusion. Based on our findings, CT imaging of the brain in patients with a clinically suspected depressed skull fracture despite any clinical neurological deficit and a GCS of 13 or more is warranted in our setting. The likelihood of injury on CT correlated inversely with the GCS.
\end{abstract}

\section{Introduction}

The Glasgow Coma Scale (GCS), introduced by Teasdale and Jenner in 1974, was devised to provide a uniform approach to the clinical assessment of patients with acute head trauma. The scale measures levels of arousal and awareness and attempts to assign numeric values (1 to 5 ) to eye opening, the best motor response and the best verbal response. As a predictor of outcome, scores of 13 to 15 are considered to correspond with mild injury, 9 to 12 with moderate injury and 8 or lower with severe injury. $^{1-3}$

Since the advent of computed tomography (CT) scans in the early 1970s, the demand from emergency room physicians for CT scans for minor head injury patients is a routine practice, although most of these investigations are normal and fewer than $1 \%$ of patients require neurosurgery. ${ }^{4,5}$ It is not known how many CT scans are done in South Africa but, according to one estimate in the USA, even a $10 \%$ reduction in the number of CT scans in patients with minor head injuries would save more than $\$ 20$ million per year. ${ }^{6}$

Over the past decade, numerous studies have investigated and attempted to regulate the utility of head CT in patients with minor head injury, such as the New Orleans criteria, the Canadian CT head rule and the National Institute for Health and Clinical Excellence (NICE) head injury guidelines. These studies use lists of clinical findings, any of which call for CT after minor head injury, ${ }^{7}$ and have given rise to a good deal of controversy. ${ }^{8}$ The aim of our study was to carry out a retrospective analysis of CT scans performed in the Radiology Department at Pelonomi Hospital in Bloemfontein. Only patients with clinically diagnosed depressed skull fracture with a GCS of 13 - 15 were included in this study and where the presence of brain pathology on CT, if any, was documented. The diagnosis of a depressed skull fracture was made by clinical examination of the patient's head and a skull X-ray to confirm the clinical observation. By undertaking this study, we hoped to provide more reliable guidelines permitting general practitioners, specialists and radiologists to be more selective in using CT scans, without compromising the care of patients with minor head injuries.

\section{Methods}

A retrospective descriptive analysis was conducted at the Pelonomi Hospital Complex and trauma unit in Bloemfontein. Data from 1 January 2008 - 1 January 2009 were collected. The trauma protocol at our institution requires all patients with depressed skull fractures to undergo an acute CT brain scan, irrespective of their GCS scores. A CT examination of the brain without intravenous iodinated contrast was required for the study. All patients with a history of head trauma and depressed skull fracture on clinical examination, a GCS score of $13-15 / 15$ and no focal neurological signs referred to the Department of Radiology for an acute CT of the brain, were eligible for the study. The CT scans of patients of all age and racial groups who fulfilled the inclusion criteria were assessed.

All CT scans were performed on a GE high-speed ZXT single-slice helical CT scanner. Two radiology registrars evaluated the scans; results were verified by a specialist radiologist.

Information was also collected from patient records, and all intracranial pathology was noted. Data were captured on a form and transferred to a Microsoft Excel spreadsheet for statistical analysis of normal and abnormal findings. Approval to conduct the investigation was granted by the Ethics Committee of the Faculty of Health Sciences, University of the Free State. 
Table I. Number of patients and abnormal CT scan findings per GCS level

\begin{tabular}{|rccc|}
\hline & \multicolumn{2}{c|}{ Table I. Number of patients and abnormal CT scan findings per GCS level } \\
\hline & & & CT scan findings \\
\cline { 3 - 3 } GCS & No. of patients $(\%)$ & Normal & Abnormal \\
score & $(\boldsymbol{N = 1 3 1 )}$ & No. of patients $(\%)$ & No. of patients $(\%)$ \\
13 & $24(18.3)$ & $6(25.0)$ & $18(75.0)$ \\
14 & $28(21.4)$ & $11(39.3)$ & $21(26.6)$ \\
15 & $79(60.3)$ & $58(73.4)$ & $56(42.7)$ \\
\hline
\end{tabular}

\begin{tabular}{|llc|}
\hline \multicolumn{3}{|c|}{ Table II. Pathology groups observed on CT scan } \\
\hline & & $\begin{array}{c}\text { No. of patients } \\
(\mathbf{\%}) \\
(\mathbf{N = 1 3 1})\end{array}$ \\
No. & Pathology & $23(17.6)$ \\
1 & Cerebral oedema & $8(6.1)$ \\
& Subarachnoid & $9(6.9)$ \\
3 & haemorrhage & $22(16.8)$ \\
4 & Subdural haemorrhage & $26(19.8)$ \\
5 & Pneumocranium & $1(0.8)$ \\
6 & Intracerebral contusion/ & $7(5.3)$ \\
7 & haemorrhage & \\
& Base of skull fracture & \\
\hline
\end{tabular}

\section{Results}

Data were collected from 131 patients' records during the study period. All these patients had a clinically diagnosed depressed skull fracture and no focal neurological deficit. The GCS of the patients varied between $13 / 15$ and $15 / 15$. A CT brain scan was performed on all the patients as requested at the time of presentation. One hundred and fourteen (87.0\%) of the patients were male. Patients' ages ranged from 1 - 76 years. Fifty-six (42.7\%) of the CT brain scans showed abnormalities.

The distribution of patients with regard to GCS score and the number of abnormalities found per GCS level are shown in Table I.

Seven different groups of intracranial pathology were encountered. A number was allocated to each pathology group for statistical analysis, as shown in Table II. Three types of pathology predominated, i.e. intracerebral contusion/haemorrhage, brain oedema and pneumocranium, which were observed in more than $15 \%$ of patients overall. The other pathologies (subarachnoid, subdural and extradural haemorrhage, and base of skull fractures) occurred at a remarkably lower rate. Twenty-four (42.9\%) of the abnormal CT scans revealed a single pathology, while multiple pathologies were observed in the remainder.

\section{Discussion}

Similar to previously published reports, ${ }^{9-11}$ a considerable number of abnormal CT scans following a depressed skull fracture with no clinical focal neurological deficit were found. Although most of the injuries were minor and possibly did not require any intervention, a CT of the brain was still warranted on the basis of the intracranial pathology retrospectively identified in these patients. Furthermore, these CT scans could provide a baseline for comparison of subsequent pathology related to the initial injury in the event of later complications and patient follow-ups.

A depressed skull fracture was significantly associated with intracranial pathology, as observed by the presence of CT scan abnormality in $42.3 \%$ of patients. As shown in Table I, a clear association between incidence of intracranial pathology and GCS score was revealed, showing an increase in the rate of pathology as the GCS score decreases. Seventy-five percent of patients with a GCS score of 13 had abnormal CT scans, as opposed to $26.6 \%$ of those patients with a GCS score of 15 .

However, despite a GCS of $15 / 15$, intracranial pathology was nonetheless demonstrated. The majority $(19.8 \%)$ of these were intracerebral contusions and haemorrhages. As this study was a retrospective assessment of CT scans performed on patients fulfilling the inclusion criteria, further management of these patients with regard to observation, treatment and/or surgical intervention was beyond the scope of this investigation.

Some clinicians believe that it is necessary to identify patients with minor brain injuries. However, several problems with this approach exist. Firstly, a patient with a normal CT of the brain may have abnormal MRI scans. ${ }^{12,13}$ Secondly, an abnormal CT does not predict neurological outcome. ${ }^{14-16}$ Previous studies have shown that patients with minor brain injury requiring no surgery or medical intervention had an average stay of 1.7 days in hospital, which questions the necessity for admission. ${ }^{17}$

Some physicians ${ }^{16}$ also warn of the risk of early epidural haematomas. However, only $17(12.9 \%)$ of our patients presented with epidural haematomas. In a paediatric study, one-third of patients were diagnosed with epidural hematomas within $24-48$ hours after sustaining head injury. All of these patients presented with normal mental status. ${ }^{17}$ Patients with minor CT abnormalities and normal mental status were found to have a very low risk for deterioration. ${ }^{17}$ As a result, some centres now have a non-operative approach to asymptomatic haematomas. ${ }^{16,17}$

Regarding the results presented in Tables I and II, we are convinced that CT of the brain in patients with depressed skull fractures, GCS of 13 - 15 and no focal neurological signs, is indeed necessary, owing to the high percentage of abnormalities found in this study group.

The rate of abnormal CT findings for patients with a GCS score of 13/15, clinical depressed skull fracture and no focal neurological signs was much higher than expected. Owing to intracranial pathology observed in $42.7 \%$ of CT scans performed on patients with minor head injuries, it can be concluded that $\mathrm{CT}$ of the brain will be required in our 


\section{ORIGINAL ARTICLE}

setting for all patients with depressed skull fractures and GCS scores of 13 - 15/15 without clinical neurological deficit.

\section{Acknowledgements}

We thank Daleen Struwig (medical writer, Faculty of Health Sciences, UFS) for technical and editorial preparation of the manuscript for publication, and Professor Gina Joubert, Department of Biostatistics, Faculty of Health Sciences, UFS, for statistical analysis of the data.

1. Grossmann RI, Yousem DM. Neuroradiology: The Requisites. 2nd ed. Philadelphia: Mosby, 2003: 243-245 2. Herndon RM. Handbook of Neurologic Rating Scales. New York: Demos Vermande, 1997: 187-208.

3. Haydel MJ, Preston CA, Mills TJ, Luber S, Blaudeau E, De Blieux PM. Indication for computer tomography in patients with minor head injury. New Engl J Med 2000; 343: 100-105.

4. Peters MC. Does every minor head injury need a CT scan? Emergency Medicine News 2003; 25: 28-30.

5. Smits M, Dippel DW, de Haan GG, et al. Minor head injury: guidelines for the use of CT - a multicentre validation study. Radiology 2007; 245: 831-838.

6. Saboori M, Ahmadi J, Farajzadegan Z. Indications for brain CT scan in patients with minor head injury. Clin Neurol Neurosurg 2007; 109: 399-405.

7. Steill IG, Clement CM, Rowe BH, et al. Comparison of the Canadian CT Head Rule and the New Orleans Criteria in patients with minor head injury. JAMA 2005; 294: 1511-1518.
8. Holmes JF, Baier ME, Derlet RW. Failure of the Miller criteria to predict significant intracranial injury in patients with a Glasgow Coma Scale score of 14 after minor head trauma. Acad Emerg Med 1997; 4: 788792.

9. Stein SC, Ross SE. Mild head injury: a plea for routine early CT scanning. J Trauma 1992; 33; 11-13.

10. Harad FT, Kerstein MD. Inadequacy of bedside clinical indicators in identifying significant intracranial injury in trauma patients. J Trauma 1992: 32: 359-363.

11. Shackford SR, Wald SL, Ross SE, et al. The clinical utility of computed tomography scanning and neurologic examination in the management of patients with minor head injuries. J Trauma 1992; 33: 385394.

12. Levin HS, Williams DH, Eisenberg HM, High WM Jr, Guinto FC Jr. Serial MRI and neurobehavioural findings after mild to moderate closed head injury. J Neurol Neurosurg Psychiatr 1992; 55: 255-262.

13. Eide PK, Tysnes OB. Early and late outcome in head injury patients with radiological evidence of brain damage. Acta Neurol Scand 1992; 86: 194-198.

14. Lee ST, Lui TN. Early seizures after mild closed head injury. J Neurosurg 1992; 76: 435-439.

15. Mendelow AD, Teasdale G, Jennett B, Bryden J, Hessett C, Murray G. Risks of intracranial haematoma in head injured adults. $B M J$ 1983; 287: 1173-1176.

16. Miller EC, Derlet RW, Kinser D. Minor head trauma: is computed tomography always necessary? Ann Emerg Med 1996; 27: 290-294.

17. Knuckey NW, Gelbard S, Epstein MH. The management of "asymptomatic" epidural hematomas. J Neurosurg 1989; 70: 392-396.

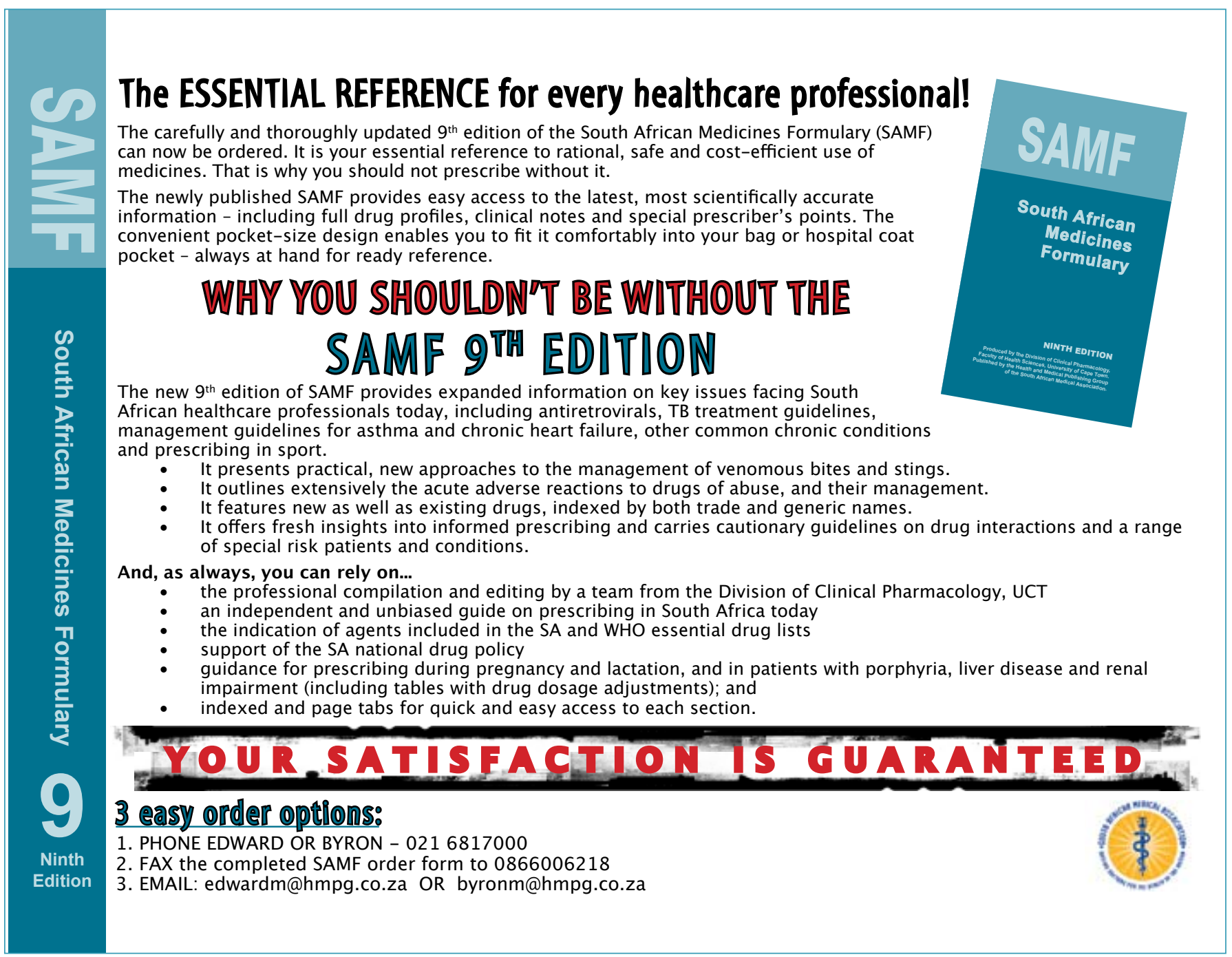

